Research Article

\title{
Road Reduction Filtering for GPS-GIS Navigation
}

\section{GEORGE TAYLOR}

Department of Geomatics

University of Newcastle upon Tyne

UK

\section{DOERTE STEUP}

Department of Geomatics

University of Newcastle upon Tyne UK

\section{GEOFFREY BLEWITT}

Nevada Bureau of Mines and Geology and Seismological Laboratory, University of Nevada, Reno

USA

\section{SIMON CORBETT}

Quality Engineering \& Survey Technology Ltd Newcastle upon Tyne UK

\section{ADRIJANA CAR \\ Department of Geomatics \\ University of Newcastle upon Tyne \\ UK}

\section{Key words}

GIS, GPS, digital mapping, map matching, vehicle navigation.

\section{Short title}

Road Reduction Filtering 
Research Article

\title{
Road Reduction Filtering for GPS-GIS Navigation
}

\author{
GEORGE TAYLOR \\ Department Of Geomatics \\ University of Newcastle upon Tyne
}

\section{DOERTE STEUP}

Department of Geomatics

University of Newcastle upon Tyne

\author{
GEOFFREY BLEWITT \\ Nevada Bureau of Mines \\ and Geology and Seismological Laboratory \\ University of Nevada
}

SIMON CORBETT

Quality Engineering \& Survey Technology Ltd

\author{
ADRIJANA CAR \\ Department of Geomatics \\ University of Newcastle upon Tyne
}

\begin{abstract}
A novel method of map matching using the Global Positioning System (GPS) has been developed for civilian use, which uses digital mapping data to infer the $<100$ metres systematic position errors which result largely from "selective availability" (S/A) imposed by the U.S. military. The system tracks a vehicle on all possible roads (road centre-lines) in a computed error region, then uses a method of rapidly detecting inappropriate road centre-lines from the set of all those possible. This is called the Road Reduction Filter (RRF) algorithm. Point positioning is computed using C/A code pseudorange measurements direct from a GPS receiver. The least squares estimation is performed in the software developed for the experiment described in this paper. Virtual differential GPS (VDGPS) corrections are computed and used from a vehicle's previous positions, thus providing an autonomous alternative to DGPS for in-car navigation and fleet management. Height aiding is used to augment the solution and reduce the number of satellites required for a position solution. Ordnance Survey (OS) digital map data was used for the experiment, i.e. OSCAR $1 \mathrm{~m}$ resolution road centre-line geometry and Land Form PANORAMA 1:50,000, 50m-grid digital terrain model (DTM).

Testing of the algorithm is reported and results are analysed. Vehicle positions provided by RRF are compared with the "true" position determined using high precision (cm) GPS carrier phase techniques. It is shown that height aiding using a DTM and the RRF significantly improve the accuracy of position provided by inexpensive single frequency GPS receivers.
\end{abstract}

\section{INTRODUCTION}

The accurate location of a vehicle on a highway network model is fundamental to any in-car-navigation system, personal navigation assistant, fleet management system, National Mayday System (Carstensen, 1998) and many other applications that provide a current vehicle location, a digital map and perhaps directions or route guidance. A great 
many of these systems use the Global Positioning System (GPS) to initially determine the position of a vehicle.

The Global Positioning System has become the most extensively used positioning and navigation tool in the world. GPS provides civilian users with an instant (real-time) absolute horizontal positional accuracy of approximately 100 metres. Most of this error is due to intentional dithering of the GPS timing signal by the US Department of Defence, an effect known as Selective Availability (S/A). This level of positional accuracy is insufficient to ensure that a vehicle's location will correspond with the digitally mapped road on which the vehicle is travelling.

A number of methods have been successfully developed to significantly improve GPS accuracy, the most notable being differential GPS (DGPS). In DGPS two receivers work together, one knows its exact position, monitors the errors in its position as provided by GPS, and transmits corrections for these errors to the other receiver. Realtime DGPS can improve positional accuracy down to 1 to $5 \mathrm{~m}$. However, the use of real-time DGPS in a moving vehicle requires additional data in the form of pseudorange corrections, i.e. computed errors in the satellite range measurements. Continuous reception of terrestrial radio transmissions or communication satellite broadcast is required to receive these corrections.

Often data can be combined from multiple sources integrating GPS with other navigational tools, attitude sensors such as the gyrocompass, vehicle odometer, flux gate compass and other dead reckoning methods. This use of multiple data sources again helps to correct for the error (noise) on the GPS position output. Multiple sensor data integration algorithms for vehicles are discussed by Mattos (1993). Dead reckoning produces the observed track by adding together the position vectors received from the sensor processor (Collier, 1990).

The fact that vehicles are generally constrained to a finite network of roads provides computer algorithms with digital information that can be used to correlate the computed vehicle location with the road network. This is known as map matching. Many methods have been devised for map matching (Scott, 1994) (Mallet et al., 1995). Our research has developed and tested an algorithm that utilizes GPS for the initial vehicle position and geometric information, computed from the digital road network itself, as the only other source of data for map-matching.

This paper is organized as follows; an overview of map matching techniques is given, the new methods developed in this project are described i.e. the Road Reduction Filter (RRF) and Virtual DGPS (VDGPS) computation, concluding with a report on the testing and evaluation of the methodology.

\section{MAP-MATCHING METHODOLOGIES}

Map-matching techniques vary from those using simple point data, integrated with optical gyro and velocity sensors (Kim, 1996), to those using complex mathematical techniques such as Kalman Filters (Tanaka et al., 1990).

A semi deterministic map-matching algorithm, described by French (1997), assumes 
that the vehicle is always on a predefined route or road network. The algorithm determines where the vehicle is along a route or within the network by determining instantaneous direction of travel and cumulative distance. This is a dead reckoning system, driven by interrupts from differential odometer sensors installed on the left and right wheels. The system uses the digital road map to check for correct left or right turns and to remove distance measurement. The positional error is converted into along-track and cross-track errors, allocating the first to the distance sensor and the second to the heading sensor errors (Mattos, 1993). For example, if the sensors indicate a 90-degree left turn and the digital mapping confirms this with the vehicle's current position, the distance count may be reset to zero. Dead reckoning and map-matching systems like this are often linked with GPS receivers through software filtering schemes such as Kalman filtering (Levy, 1997).

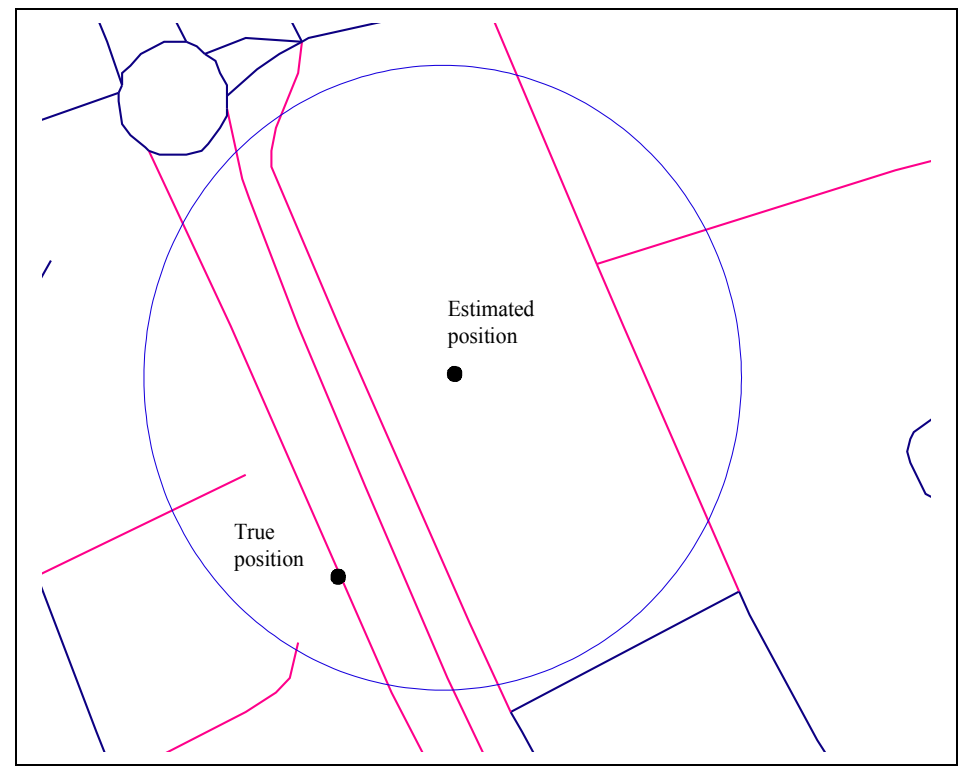

Figure 1. Potential road centre-lines

Scott (1994) provides a mathematical framework for map matching of vehicle positions using GPS. In this framework, the theoretical performance of a map-aided estimation process is assessed using error statistics to translate the raw positions onto the road network. However, Scott acknowledges that a key component of the map-aided estimator is correct road identification. All performance measures derived for the estimator are not applicable if the vehicle position has been projected onto the wrong road. This is true for performance measures of any map-matching algorithm.

Systems that use only geometric information must utilize the "shape" of line segments (road centre-lines) that define the road network (Bernstein et al., 1998). A logical first step is to determine which road centre-lines are candidates for the vehicle's true location. All road centre-lines that cross the region of the possible true position must be located. For example, there are eight potential centre-lines, highlighted in red, within the $80 \mathrm{~m}$ region displayed in figure 1 . This region will vary from a $100 \mathrm{~m}$-radius circle with centre at the computed raw/uncorrected GPS point position, (Estimated position in figure 1) to a small error ellipse centred on the corrected position, with typical semi- 
major and semi-minor axis of $5 \mathrm{~m}$ and $3 \mathrm{~m}$ respectively. The shortest Euclidean distance from the GPS position to each of these road segments is computed and ordered by decreasing distance. The method used here must first calculate coefficients $A, B$ and $C$ for the implicit and normalized equation of a line through two points that define the road centre-line (line segment):

$$
A x+B y+C=0
$$

Let the line be described as going from point $k$ to $l$. If coordinates $l_{E}, l_{N}$ are grid Easting and Northing for point $l$, and $k_{E}, k_{N}$ are Easting and Northing for point $k$, then:

$$
\begin{aligned}
& A=k_{N}-l_{N} \\
& B=l_{E}-k_{E} \\
& C=k_{E} l_{N}-l_{E} k_{N}
\end{aligned}
$$

If $A$ and $B$ are both zero then it is a bad line definition, otherwise

$$
\begin{aligned}
& A=A / \sqrt{A^{2}+B^{2}} \\
& B=B / \sqrt{A^{2}+B^{2}} \\
& C=C / \sqrt{A^{2}+B^{2}}
\end{aligned}
$$

Then calculate the shortest distance $D$ from the GPS position $p$ to line $A x+B y+C=0$

$$
D=\left|A p_{E}+B p_{N}+C\right|
$$

If $D$ is equal to zero, then $p$ is on the line. In addition, if $D$ is positive, $p$ is to the right of the line, or if $D$ is negative, $p$ is to the left of the line joining $k$ to $l$. This information is of use to map-matching algorithms, and is used here.

It is not simply a matter of finding the line segment nearest to position $p$. This will often give an incorrect result. For example, in figure 1, the vehicle is on the highlighted road segment in the SW corner, but this is not the nearest road line segment to the position $p$. Given a single GPS point position for the vehicle, it is not possible to determine which is the correct road segment, if there is more than one road segment in the neighbourhood of the possible true position.

A better way to proceed is to match arcs defined by a series of GPS point positions $\left\{p_{1}\right.$, $\left.p_{2}, p_{3} \ldots \ldots, p_{\mathrm{n}}\right\}$ with an arc defined by a set of points that define a partial road centre-line $\left\{c_{1}, c_{2}, c_{3} \ldots \ldots, c_{\mathrm{n}}\right\}$. One method used for matching two curves (arcs) is to use the distance between them.

If $\mathrm{P}$ and $\mathrm{C}$ are two such arcs, the minimum distance between any pair of points taken from each arc, i.e, may define the shortest distance between them.

$$
\|\mathbf{P}-\mathrm{C}\|_{\min }=\min _{p \in P, c \in C}\|p-c\|
$$

Bernstein et al. (1998) describes a curve (arc) matching algorithm, which uses a measure of the average distance between two arcs. This algorithm is implemented for 
map matching in Princeton University's Large-Scale Automobile Routing (PULSAR) Project. Arcs must be parameterised by using a function such as $p:[0,1] \rightarrow \mathrm{P}$, then

$$
\|P-C\|=\int_{0}^{t}\|p(t)-c(t)\| d t
$$

The problem with this algorithm is that it will only reliably detect the best match for arcs of the same length, which limits its ability to identify the correct arc in certain circumstances, e.g. slow moving vehicles.

Moments and moment invariants may potentially be used to match arcs. These properties of shapes are used in digital pattern recognition since they are independent of general linear transformations. Singer (1993) describes a method of moment expansion for linear objects (arcs), which may be used, for arc comparisons. The moments associated with a line segment $l i$ can be written as

$$
\mu(r, s)=\int_{l i} x^{r} y^{s} d l
$$

Appropriate moments of two matching arcs would differ only by some small predetermined tolerance.

Other methods are also used to reduce the number of potential road segments for the correct vehicle position. The topology of the road network may also be used. If the length of the connected route through a network from the present position on one particular road segment to the next position on another potential road segment is outside the possible range of distance travelled so far, that potential road segment is rejected. Carstensen (1998) has looked at the effects of filtering autonomous GPS points by number of satellites tracked, Dilution of Position (DOP) values or satellite geometry, and velocity and acceleration of the vehicle between positions. Filtering of potential road segments may be achieved using some of these measures and other criteria such as distance travelled and change of heading between vehicle positions, both criteria are used here. 


\section{RESEARCH METHODOLOGY}

The method of map matching developed in this project is dependent on two main innovative techniques.

1. The computation algorithm of a least-squares estimation of the position, described for example by Blewitt (1997), provides complete control over which satellites will be used in the solution. This avoids step functions in the GPS positions as a result of the loss and gain of satellites. It enables the use of height aiding in the solution, i.e. removes one of the unknown parameters, thus one less satellite is required for the computation. Furthermore, it also enables the calculation and use of pseudorange corrections derived from the digital road network data virtual DGPS (VDGPS).

2. A method of modelling Selective Availability (S/A) is currently being developed. Although in the long term S/A introduced error will reduce to approximately a Gaussian distribution (random error), in the short term (20 seconds) the effect of S/A can be viewed as a slowly varying bias (Scott, 1994). S/A will move the point position of a stationary receiver by approximately $10 \mathrm{~m}$ to $30 \mathrm{~m}$ per minute. S/A modelling using map matching will be reported when test results are undertaken and analysed.

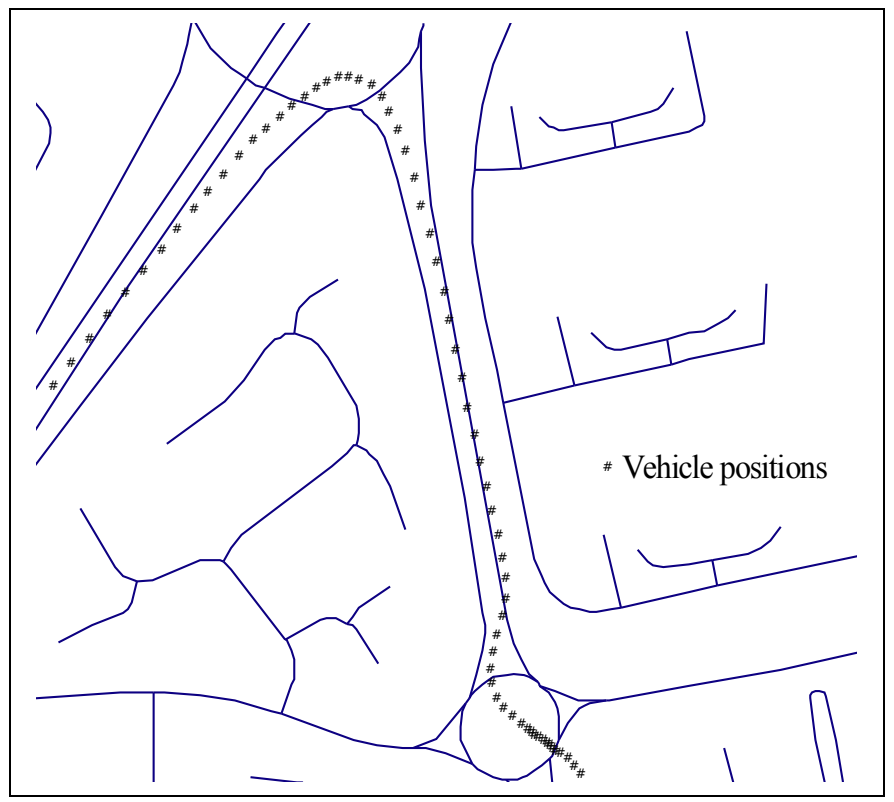

Figure 2. Raw GPS positions and road centre lines 


\section{ROAD REDUCTION FILTER (RRF) MAP MATCHING ALGORITHM}

The general approach adopted in this work to improve the accuracy of the computed position of a vehicle is to identify all possible candidates for the correct road and systematically remove the wrong ones. This is achieved by map matching; all candidate road segment arcs are compared with the trajectory described by the Raw (uncorrected) point positions. This is based on the distance travelled and bearing of the Raw point positions compared with the corresponding Ref positions on road centrelines. The algorithm is as follows:

1. A Raw vehicle position is computed using all satellites available plus height aiding, where height is obtained from a DTM, which used to provide an extra equation in the least squares approximation computation, i.e. computation with a minimum of three satellites. For the first epoch all roads (road centre-line segments), which are within $100 \mathrm{~m}$ of the computed Raw position are selected. We can guarantee with $95 \%$ confidence that the vehicle is on one of these road segments, according to GPS specification, (Department of Defence, 1992). The point on each of the $n$ road segments that computes the shortest distance to the Raw position, using equations (1) to (4), is selected as the first approximation of the true location of the vehicle, its Ref position. That is, we have $n$ Ref positions that we can use to generate virtual DGPS corrections for use with the next epoch's computed Raw position.

2. Virtual DGPS corrections for each satellite pseudorange are computed at each of the $n$ Ref positions on each road segment for the current epoch, giving $n$ different sets of virtual DGPS corrections.

3. The next epoch Raw position is computed, as in step 1.

4. Each of the virtual DGPS corrections (step 2) are added to the Raw position (step 3) to give $n$ Cor positions for each $n$ road segments.

5. Each of these $n$ Cor positions is now snapped back onto the nearest road-centre lines to give $n$ Ref positions. Go to step 2 .

Steps 2 to 5 are repeated continuously. The output point position from the RRF is either taken from the only remaining road centre-line or is the weighted mean of points on all candidate road centre-lines. The weighted mean position is calculated as follows:

$$
\begin{aligned}
& \text { Easting }=\frac{\sum \frac{\text { EREF }}{\text { Average Bearing error }}}{\sum \frac{1}{\text { Average Bearing error }}} \\
& \text { Northing }=\frac{\sum \frac{\text { NREF }}{\text { Average Bearing error }}}{\sum \frac{1}{\text { Average Bearing error }}}
\end{aligned}
$$

Where EREF and NREF are Easting and Northing for each of the $n$ Ref positions. These are inversely weighted by the Average Bearing error (described below), which positions the vehicle closer to the road with the smallest bearing error. This helps to smooth point positions when the one correct road centre-line has not yet been found. When the 
correct one is found only that one is processed, until a road junction is encountered, when again as in step 1, a number of road centre-lines that cross the computed error region would have to be considered. The computed error region is an error ellipse calculated from the elements of the cofactor matrix for the estimated parameters in the least squares computation of the position. The observation error variance used to scale this ellipse is dependent on of the Distance error and Bearing error (described below), i.e. the larger these errors the larger the dimensions of the error ellipse.

This process from steps 2 to 5 is repeated for each new epoch. At each epoch for each of the $n$ road segments the following data is computed and stored:

$\begin{array}{lll}\text { Raw distance } & - & \text { from previous Raw to current Raw position. } \\ \text { Ref distance } & - & \text { from previous Ref to current Ref position. } \\ \text { Raw bearing } & - & \text { from previous Raw to current Raw position. } \\ \text { Ref bearing } & - & \text { from previous Ref to current } \text { Ref position. }\end{array}$

This data is held for the last 20 one second epochs for each road centre line processed.

\section{DETERMINING THE CORRECT ROAD CENTRE-LINE}

The task of the RRF is to determine the correct road centre-line segment from the set of those possible, or conversely, which road centre-lines to reject. The trajectory defined by Raw GPS positions computed from observations taken by a receiver in a moving vehicle is correlated with the shape of the digitised road centre-line on which the vehicle is travelling, see figure 2. This correlation is high if the vehicle is travelling at high speed, and low if the vehicle is travelling at low speed (5MPH or $2.24 \mathrm{~m} / \mathrm{s})$, because at high speed the S/A bias changes less per unit distance travelled.

By calculating values for distance travelled and bearing between epochs for Raw positions and comparing these values with equivalent Ref positions, it is possible to filter out many incorrect road centre-lines. There is a very high correlation if we compare bearing and distance between successive Raw positions and the bearing and distance between successive Ref positions on the correct road centre-line, if the vehicle is moving. We can calculate the following errors for each epoch:
1. Distance error
$=$ absolute value of the difference between Raw
2. Bearing error = absolute value of the difference between Raw distance and Ref distance bearing and Ref bearing
3. Average Bearing error $=$ average of 2 (Bearing errors) for the last 20 positions 
Figures 3 and 4 compare errors for eight different series of Ref positions, i.e., eight different road centre-lines, over a period of 20 epochs (1 second interval). Figure 3 displays the distance errors and figure 4 the bearing errors. The bearing errors are clipped at 90 degrees to provide a graph at a scale that is legible. The filter is switched off. If a pre-defined tolerance value is set, perhaps $5 \mathrm{~m}$, it can be seen in figure 3 that series 2, 6, 7 and 8 can quickly be filtered out at almost any epoch. Series 3 and 4 are not as easy to identify for removal, and series 1 is even more difficult to remove. Series 5 is the set of Ref positions on the correct road centre-line, this has a maximum error of $5 \mathrm{~m}$ at epoch 10 , where the vehicle is driven through a roundabout, see figure 1 . Series $3,4,1$ and 5 are in fact positions from the four parallel road centre-lines displayed in red in figure 1. Similarly for the bearing errors in figure 4, a filter value of 30 degrees would remove all but the correct series of Ref positions. The Raw, Cor and Ref positions for this correct road centre-line (series 5) are shown in figure 5.

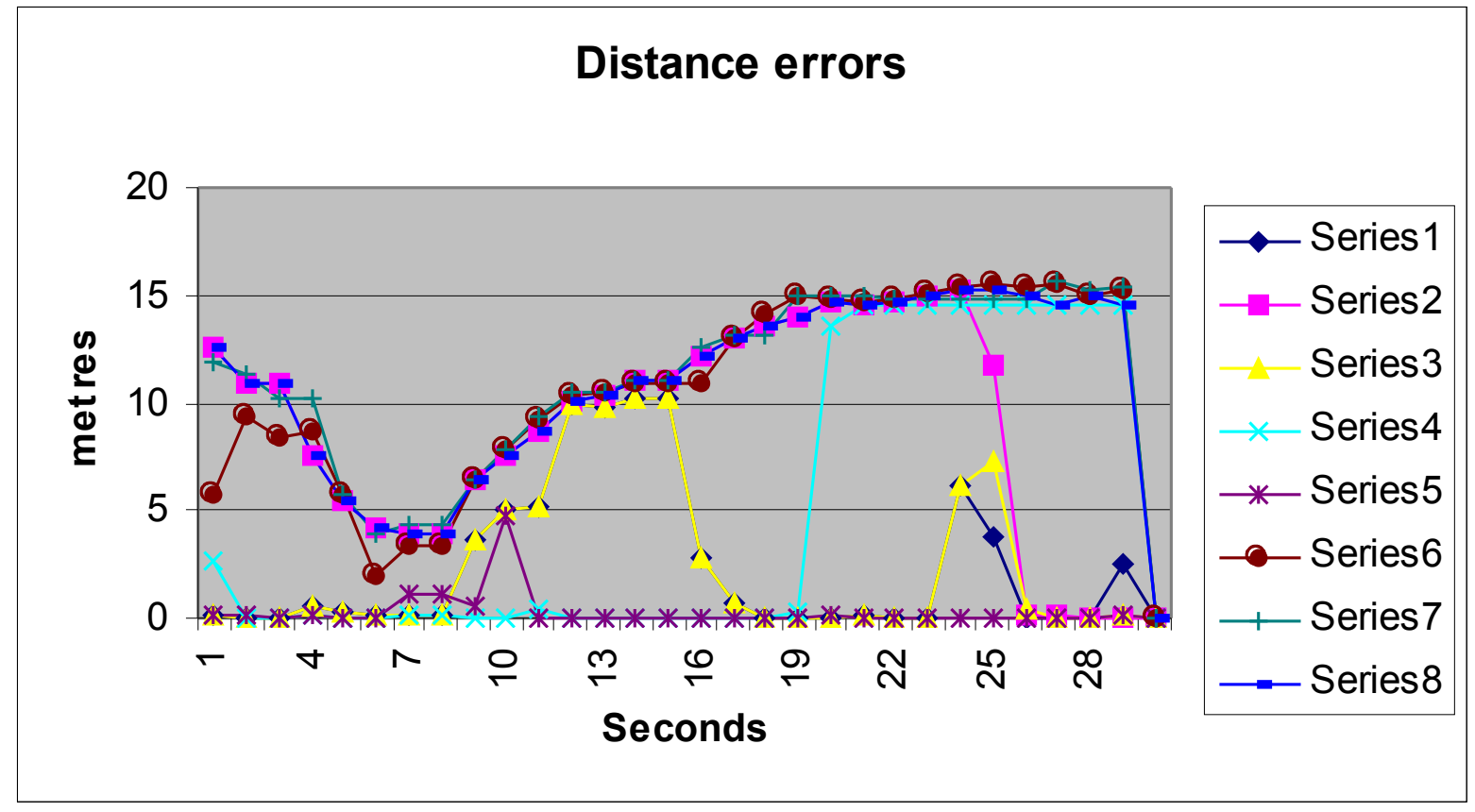

Figure 3. Distance errors for tracked road centre-lines 


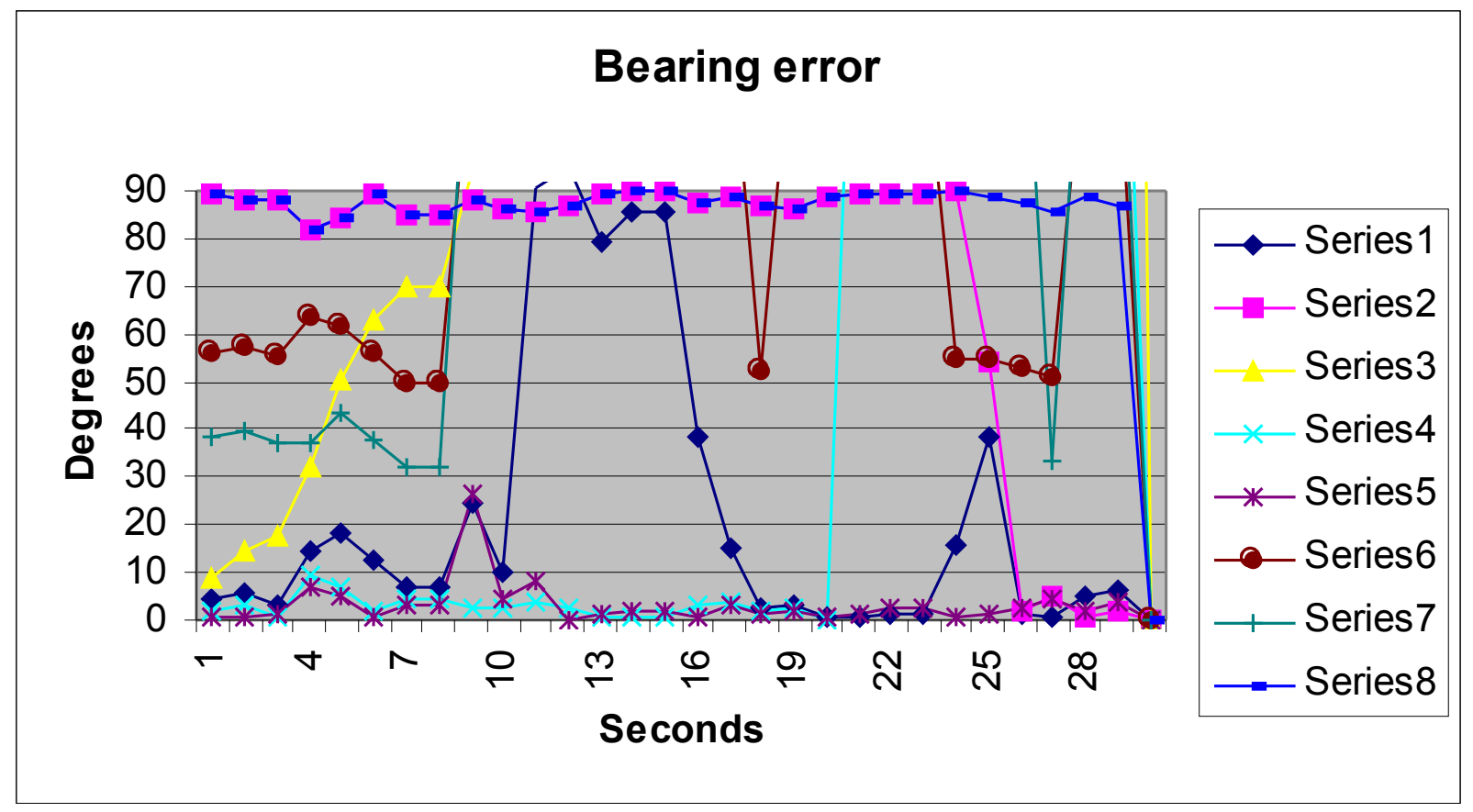

Figure 4. Bearing errors for tracked road centre-lines

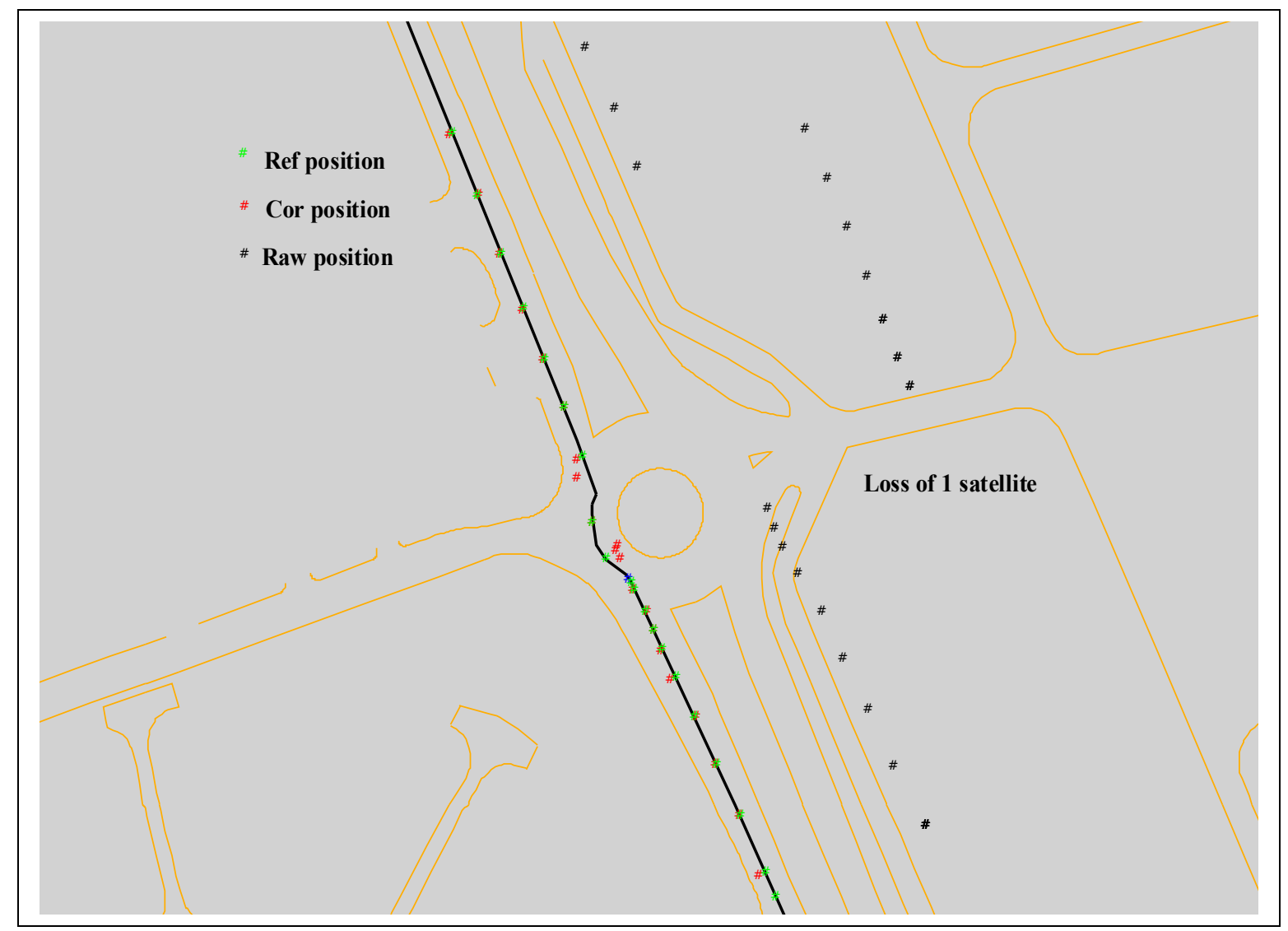

Figure 5. Correct road centre-line only 


\section{TESTING VDGPS}

To evaluate the performance of the algorithm, data was collected in a vehicle driven on roads in the suburbs of Newcastle upon Tyne, UK. An Ashtech Z12 receiver was used in the test vehicle with an Ashtech 700718B Geo.III L1/L2 antenna mounted on the roof. GPS data was recorded at one-second intervals. Over the same period, a static Ashtech Z12 receiver recorded base station data on the roof of the Department of Geomatics, University of Newcastle. The data sets were then post processed a number of times, using:

1. Only software developed during this project, using C/A code observations from the vehicle and the OS digital map and DTM data.

2. GPSurvey software using dual frequency phase data from both the vehicle and the base station to compute a high precision (cm accuracy) GPS solution, i.e. Real Time Kinematic (RTK) processing.

Both methodologies have used the following common settings, 15-degree elevation mask, Saatsamoinen tropospheric model and no cut off value for Position Dilution of Precision (PDOP). DOP modifies the ranging error to a satellite, and it is caused solely by the geometry between the user and the set of satellites.

The computed point position outputs from method 1 are; uncorrected GPS receiver positions (RAW), uncorrected GPS receiver positions using OS DTM data for height aiding (RAW+HA) and RRF positions using both OS road centre-line data and OS DTM data for height aiding (VDGPS). To correctly display the vehicle positions on OS large scale mapping all resultant latitude, longitude and height coordinates from methods 1 and 2 were transformed from WGS84 (World Geodetic System, 1984) to OSGB36 (Ordnance Survey of Great Britain, 1936) National Grid. This was computed using the Ordnance Survey's OSTN97 with a nominal transformation accuracy of $20 \mathrm{~cm}$ with respect to OS primary and secondary triangulation networks, and the European Terrestrial Reference Frame 89 (ETRF89) realization of WGS84. To use height aiding in the least squares solution the Ordnance Datum Newlyn orthometric heights of the OS DTM are transformed to WGS84 ellipsoid heights using the OSGM91 geoid model, with a transformation accuracy of $10 \mathrm{~cm}$.

The "true" position of the vehicle at each epoch was assumed to be that given by the RTK solution. Comparisons are made between the following point position outputs:

- $\quad$ RTK - VDGPS

- RTK - RAW

- RTK - RAW+HA 


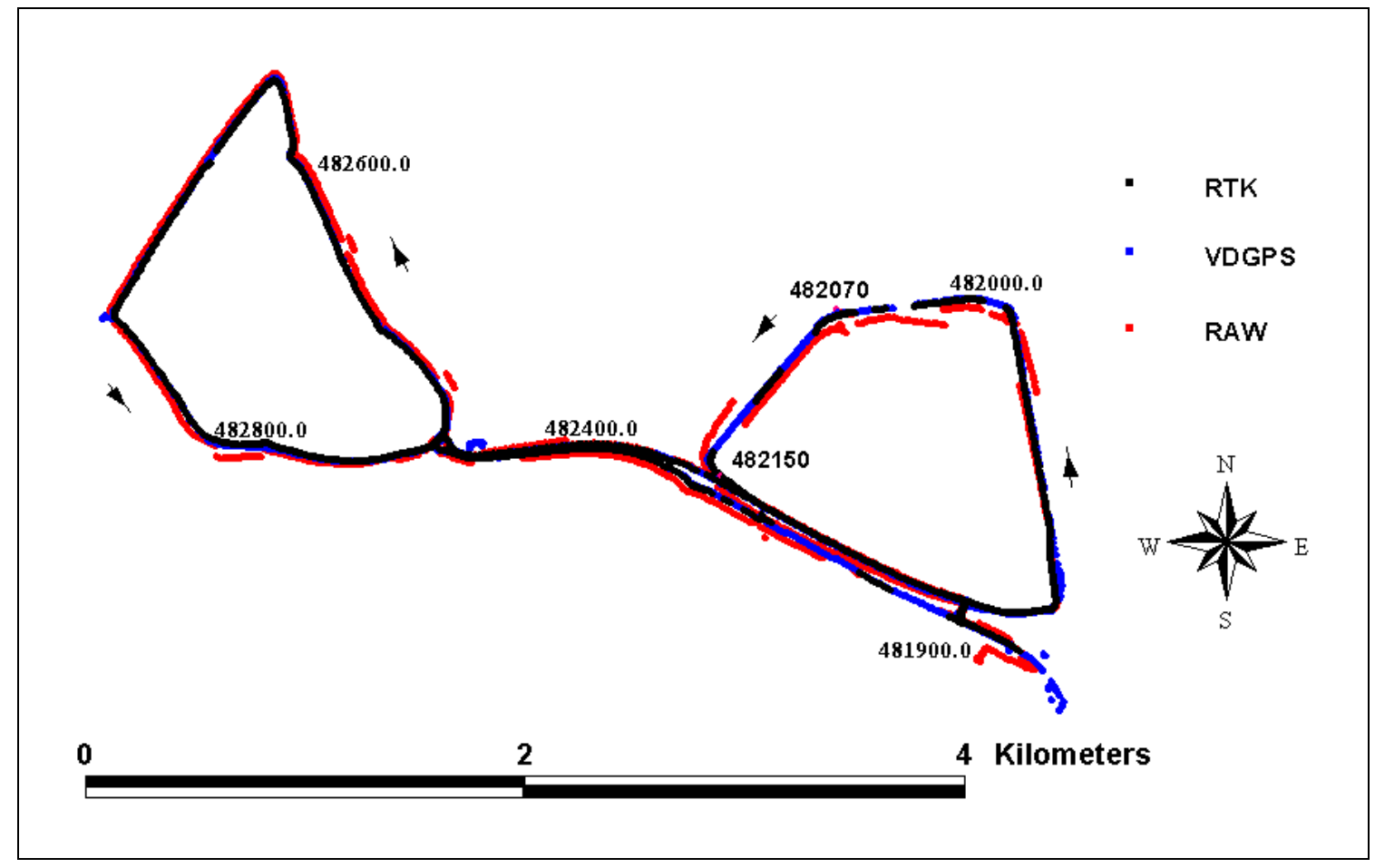

Figure 6. Test route

\section{TEST RESULTS}

Figure 6 shows the track from each of the three positioning methods. The circuit took approximately 1200 one-second epochs to complete. Table 1 summarizes the number of successfully processed epochs (1 second) for each method. All of the position fixes are counted and the total possible number of positions divides the total number of positions recorded, which is the duration of the test in seconds (Bullock, Geier et al. 1996). It can be seen that the use of height aiding, for RAW+HA and VDGPS, provide the best results as far as completeness is concerned, since a solution with one less satellite is possible.

Table 1. Processed epochs for each GPS technique

\begin{tabular}{lllcl}
\hline & RTK & RAW & RAW+HA & VDGPS \\
\hline processed epochs: & 1112 & 1230 & 1349 & 1349 \\
percentage: & $81.6 \%$ & $90.3 \%$ & $99.0 \%$ & $99.0 \%$ \\
\hline
\end{tabular}

If the RTK positions are assumed to be the truth, horizontal positional errors can be computed as follows

$$
D P=\sqrt{D E^{2}+D N^{2}}
$$

where DP, DE, DN are the position, Easting and Northing error respectively 
and

$$
\begin{aligned}
& D E=E_{R T K}-E_{i} \\
& D N=N_{R T K}-N_{i}
\end{aligned}
$$

where $E_{R T K}$ and $N_{R T K}$ are the Easting and Northing of the RTK position, and $E_{i}$ and $N_{i}$ are the corresponding positions for each of the other methods.

In order to help identify when a wrong road is selected and as an indication of how accurate the algorithm is when the correct road is isolated, the Easting and Northing errors $D E$ and $D N$ of the VDGPS method are transformed into cross track and along track errors as follows:

$$
\left(\begin{array}{l}
C_{i} \\
L_{i}
\end{array}\right)=\left(\begin{array}{cc}
\cos t_{i, i+1} & -\sin t_{i, i+1} \\
\sin t_{i, i+1} & \cos t_{i, i+1}
\end{array}\right)\left(\begin{array}{c}
D E_{i} \\
D N_{i}
\end{array}\right)
$$

where

$\mathrm{Ci} \quad$ is the cross track error of position $i$

$\mathrm{Li} \quad$ is the along track error of position $i$

$D E_{\mathrm{i}} \quad$ is the Easting error of position $i$

$D N_{i} \quad$ is the Northing error of position $i$

$t_{i, i+1} \quad$ is the bearing of the road segment between position at epoch $i$ and $i+1$

The mean, standard deviation (SD) and maximum and minimum values are also computed for each error (table 2).

Table 2: Statistics summary

\begin{tabular}{|l|r|r|r|r|r|r|}
\hline & \multicolumn{4}{|c|}{ VDGPS } & \multicolumn{1}{c|}{ RAW } & RAW+HA \\
\hline & $\begin{array}{c}\text { Cross Track } \\
\text { Error }\end{array}$ & $\begin{array}{c}\text { Along Track } \\
\text { Error }\end{array}$ & $\begin{array}{l}\text { Position } \\
\text { Error }\end{array}$ & $\begin{array}{c}\text { Position } \\
\text { Error [95\%] }\end{array}$ & $\begin{array}{c}\text { Position } \\
\text { Error }\end{array}$ & $\begin{array}{c}\text { Position } \\
\text { Error }\end{array}$ \\
\hline Mean & $-3.309 \mathrm{~m}$ & $-0.808 \mathrm{~m}$ & $14.184 \mathrm{~m}$ & $11.618 \mathrm{~m}$ & $35.727 \mathrm{~m}$ & $26.940 \mathrm{~m}$ \\
\hline $\begin{array}{l}\text { Standard } \\
\text { Deviation }\end{array}$ & $8.733 \mathrm{~m}$ & $17.548 \mathrm{~m}$ & $14.563 \mathrm{~m}$ & $9.342 \mathrm{~m}$ & $42.842 \mathrm{~m}$ & $16.603 \mathrm{~m}$ \\
\hline $\begin{array}{l}\text { Minimum } \\
\text { magnitude }\end{array}$ & $-65.970 \mathrm{~m}$ & $-74.856 \mathrm{~m}$ & $0.018 \mathrm{~m}$ & $0.018 \mathrm{~m}$ & $1.345 \mathrm{~m}$ & $2.367 \mathrm{~m}$ \\
\hline $\begin{array}{l}\text { Maximum } \\
\text { magnitude }\end{array}$ & $28.464 \mathrm{~m}$ & $45.259 \mathrm{~m}$ & $76.272 \mathrm{~m}$ & $43.543 \mathrm{~m}$ & $272.541 \mathrm{~m}$ & $91.898 \mathrm{~m}$ \\
\hline
\end{tabular}

A summary of the test results, calculated using equations 9,10 and 11 , are shown in table 2. The Position Error [95\%] statistics are those of the VDGPS with the largest 5\% of Position errors removed. The [95\%] statistics have much lower values for mean, SD and maximum error, which demonstrates that there are a few very large errors (over 
$50 \mathrm{~m}$ ) in the full set of VDGPS results, this is shown in figure 7. Figure 10 displays the length of road centre-line on which almost all of these large errors arise, i.e. between epochs 482070 and 482150 . The only other time such large errors occur is at the very start of the observation period. In fact, these are both good examples of where the RRF is least effective, when the vehicle is stationary and for a short period after that. That is, before the vehicle moves off at the start of the observations and for approximately 20 seconds at a set of traffic lights. It can be seen from figure 6 and 10 that as soon as the road turns, at epoch 482155 approx. this large position error is rapidly reduced.

The main interest here is the comparison of positions provided by VDGPS and those provided by a single GPS receiver with no corrections (RAW). Note that cross track and along track errors given in table 2 are those of the full set of VDGPS positions. The worst cross track errors are again between epochs 482070 and 482150, in fact at epoch 482090 , figure 11, when the vehicle is stationary and the wrong road has been selected by the RRF. The along track errors, figure 12 , are also at a maximum of $-80 \mathrm{~m}$ near epoch 482090, meaning the car is positioned $80 \mathrm{~m}$ behind where it should be on the road. Figure 12 also displays how the along track error gradually decreases as the algorithm derived VDGPS positions catch up with the with the true vehicle positions on the road. It is clear from table 2 that VDGPS provides much reduced position error than RAW, producing an improvement from a mean position error of $36 \mathrm{~m}$ down to $14 \mathrm{~m}$, compare the distributions of position errors in figures 7 and 9. Both the range of error and SD are also reduced. The very high position errors in RAW $(>200 \mathrm{~m})$, shown in figure 9, are a result of not applying any cut off value for PDOP, this is done to maximize the possible number of epochs for which a position may be computed. If the worst 5\% position errors are removed from VDGPS, the results are further improved, see table 2. Figures 8 show the distribution of position errors for VDGPS at $95 \%$ confidence interval.

The increasing frequencies of errors between $16 \mathrm{~m}$ and $28 \mathrm{~m}$ for VDGPS are due to the temporary inability of the RRF to identify the correct road, i.e. for short periods an incorrect road centre-line is being tracked. Cross track errors are only significantly large again when temporarily the correct road centre-line has not been isolated and a weighted mean position is used, as described in equation 8 . 


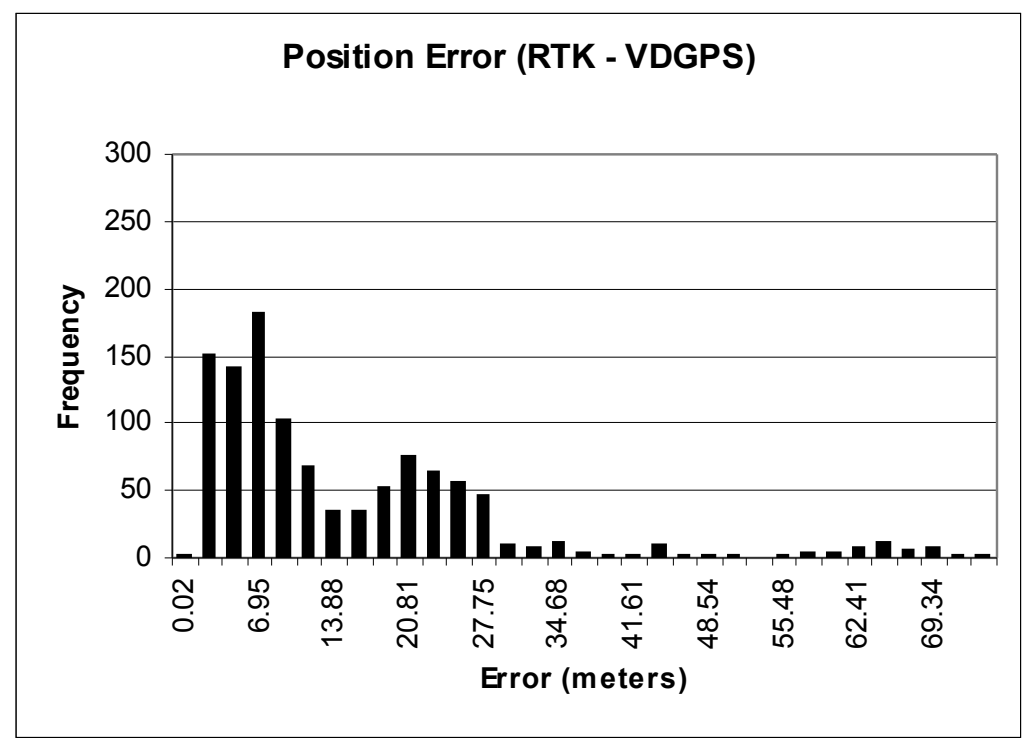

Figure 7. VDGPS Position Error distribution

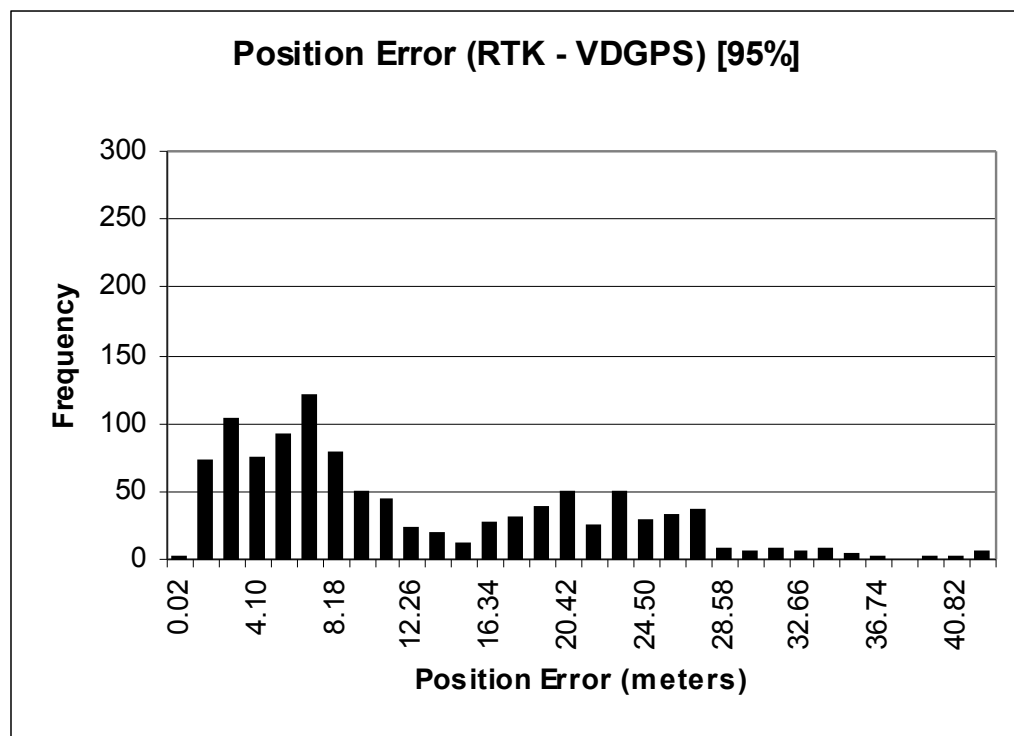

Figure 8. VDGPS Position Error distribution, 95\% confidence interval 


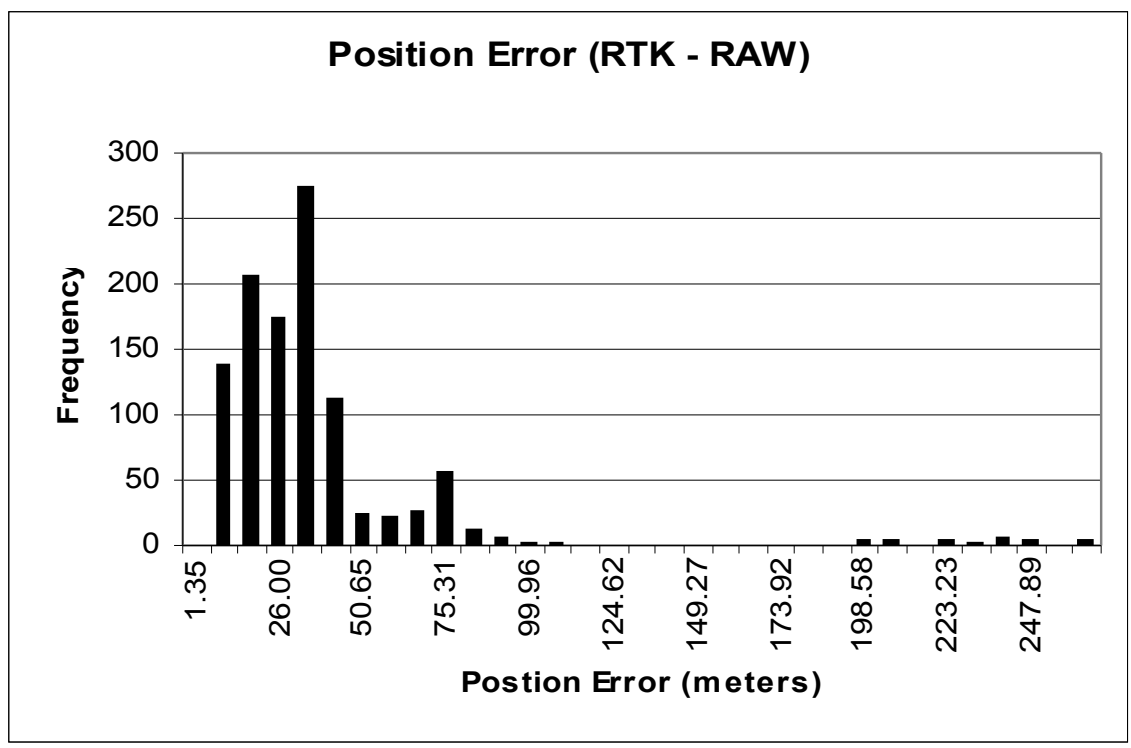

Figure 9. RAW Position Error distribution

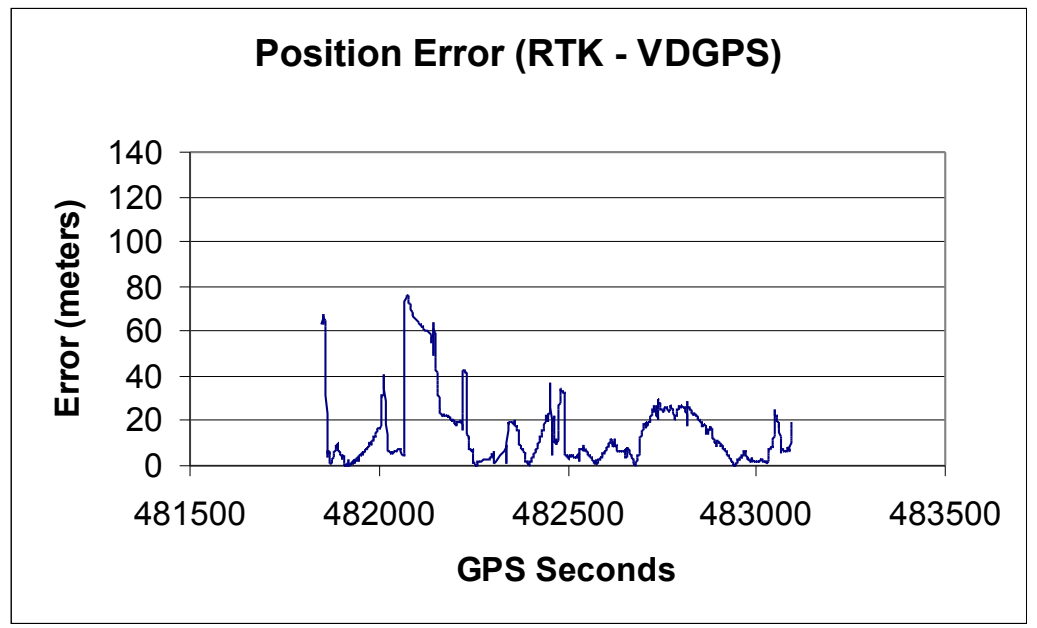

Figure 10. VDGPS Position Errors 


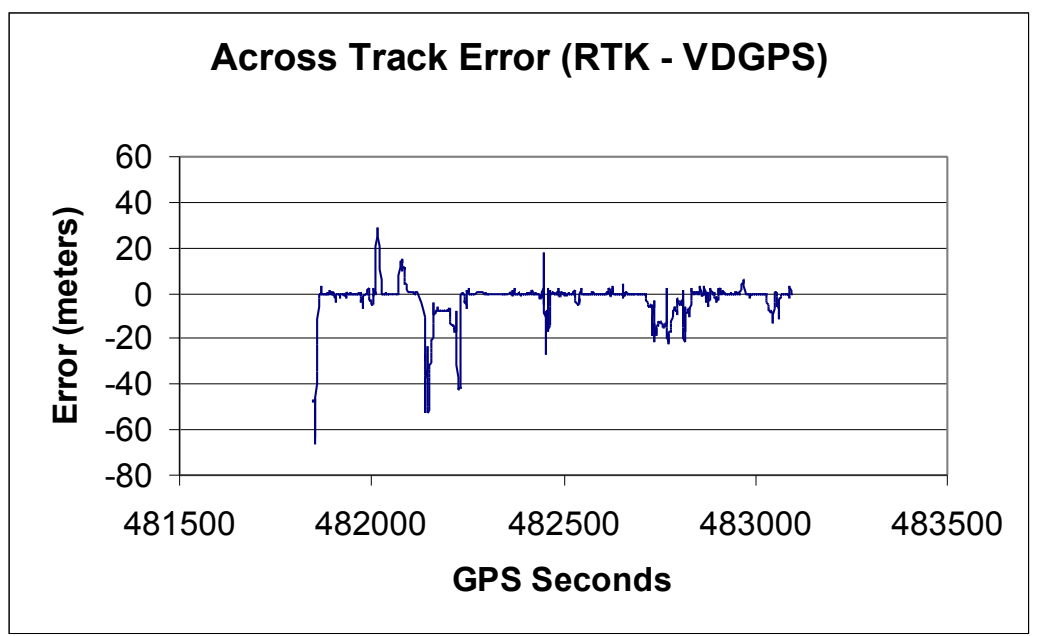

Figure 11. Cross track errors

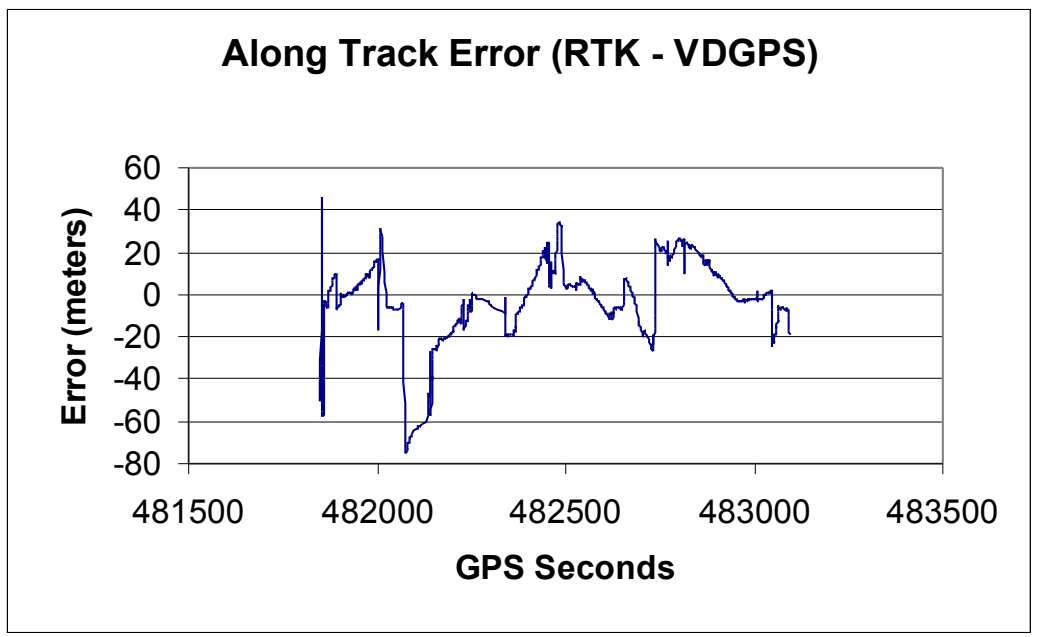

Figure 12 .Along track errors

\section{CONCLUSIONS}

The augmentation of GPS code data with height and road network information, obtained from digital mapping, for the positioning of a moving vehicle has improved reliability by almost $10 \%$, i.e. $90.3 \%$ to $99 \%$ of all possible epochs processed. To put it another way, VDGPS reduces the number of positions that cannot be computed from around $10 \%$ of all epochs to $1 \%$ of all epochs, an improvement of approximately $90 \%$. The mean horizontal position accuracy has changed for the better from $35.727 \mathrm{~m}$ to $14.184 \mathrm{~m}$, with good improvements in standard deviation and range of errors, see table 2. If gross errors are removed, these improvements are further enhanced.

This experiment has successfully integrated only satellite navigation and digital mapping data to improve accuracy and reliability of the estimated position of a moving vehicle. The computation combines raw GPS pseudorange measurements, road centre- 
line geometry and DTM data in new map matching algorithm. The work has deliberately only used geometry to improve vehicle positioning.

The use of just these two data sources has the distinct advantage of producing a completely self-contained system, requiring no radio communication for differential corrections and continuous data provision, or input from any other sensors. Furthermore, because the computation of the estimated GPS receiver position is part of the RRF and a digital terrain model derived height aiding is used in the solution, only three satellites are necessary for a solution.

One problem with the approach presented above is that Selective Availability (S/A) has horizontal errors of up to $100 \mathrm{~m}$, so while it may be possible at a particular point in time to identify the correct road, the position along the road may be in error by up to $100 \mathrm{~m}$, and is frequently plus or minus $20 \mathrm{~m}$, see along track errors in figure 12 . This along track error cannot be resolved for a straight road, but it can be resolved if the road changes direction, or if the vehicle turns a corner. Present work is focused on the development of a formal least squares estimation of Selective Availability (S/A) based on road geometry (road shape). Quality measures can be derived and used to place confidence bounds for rigorous decision-making on the reliability of such an S/A model.

Further work will include an investigation of other techniques to reduce the number of satellites required for a solution. Bullock et al. (1996), examined two satellite tracking for urban canyons when map matching requires only a two dimensional position. A test to detect a stationary receiver is being included in the RRF. The use of digital road map intelligence will be included in the RRF to improve the efficiency of isolating the correct road centre-line, e.g. one-way traffic direction, direction of travel at a roundabout, or along a dual carriageway. Furthermore, an attempt to implement hierarchical spatial reasoning techniques (Car, 1997) will be made to improve the efficiency of the RRF algorithm using road centre-line network connectivity.

\section{ACKNOWLEDGEMENTS}

This work was undertaken as part of a Royal Society Industry Fellowship and was also supported by a Department of Trade and Industry (DTI) SMART Award.

\section{REFERENCES}

Bernstein D and Kornhauser A 1998 Map matching for personal navigation assistants. In Proceedings $77^{\text {th }}$ Annual meeting, The Transport Research Board. Washington DC: $11-15$

Blewitt G 1997 Basics of the GPS Technique: Observation Equations. In Geodetic Applications of GPS, ed. B Johnson, Nordic Geodetic Commission, Sweden, ISSN 0280-5731: 10-54

Bullock B, Geier GJ, King TM and Kennedy HL 1996 Effectiveness of Two Satellite Tracking in Urban Canyon Environments. In Proceedings of ION GPS-96, Sixth International Technical Meeting of the Satellite division of The institute of Navigation. Kansas City, Missouri: 1699-1707 
Car A 1997 Hierarchical Spatial Reasoning: Theoretical Consideration and its Application to Modelling Wayfinding. ed. A. U. Frank and P. Haunold, Geoinfo Series. Vienna, Austria. ISBN 3-901716-10-6

Carstensen Jr LW 1998 GPS and GIS: Enhanced Accuracy in Map Matching through Effective Filtering of Autonomous GPS Points. Cartography and Geographical Information Systems 25: (1) 51-62

Collier C 1990 In-vehicle route guidance systems using map matched dead reckoning. In Proceedings IEEE Position and Navigation Symposium: 359-363

Department of Defense and Department of Transportation 1992 Federal Radionavigation Plan. USA Government

French RL 1997 Land Vehicle Navigation and tracking. Global Positioning System: Theory and Applications, Volume II: 275-301

Kim JS, Lee JH, Kang TH, Lee WY and Kim YG 1996 Node based map matching algorithm for car navigation system. In Proceedings of the 29th ISATA Symposium, Florence 10: 121-126

Levy LJ 1997 The Kalman Filter: navigation's Integration Workhorse. GPS World, 8: (9) 65-71

Mallet P and Aubry P 1995 A low-cost localisation system based on map matching technique. In Proceedings of the International Conference on Intelligent Autonomous Systems, Karlsruhe, Germany: 72-77

McCall R B 1970 Fundamental Statistics for Psychology. Harcourt, Brace \& World, Inc., ISBN 0-15-529410-5

Mattos PG 1993 Intelligent Sensor Integration Algorithms for Vehicles. In Proceedings of ION GPS-93, Sixth International Technical Meeting of the Satellite division of The institute of Navigation, Salt Lake City, Utah: 1591-1597

Scott C 1994 Improving GPS Positioning for Motor-Vehicle through map matching. In Proceedings of ION GPS-94, Seventh International Technical Meeting of the Satellite division of The institute of Navigation, Salt Lake City, Utah: 1391-140

Singer MH 1993 A general approach to moment calculations for polygons and line segments. Pattern Recognition 26: (7) 1019-1028

Tanaka J, Hirano K, Itoh T, Nobuta H and Tsunoda S 1990 Navigation System with Map-Matching Method. Proceedings of the SAE International Congress and Exposition: 45-50 